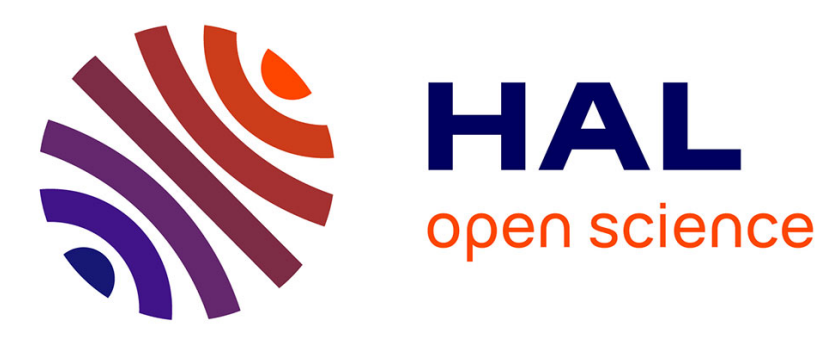

\title{
Étude théorique des spectres optiques des ions Pr3+ dans AlLaO 3
}

\author{
N. Pelletier-Allard, F. Martin-Brunetière
}

\section{To cite this version:}

N. Pelletier-Allard, F. Martin-Brunetière. Étude théorique des spectres optiques des ions Pr3+ dans AlLaO 3. Journal de Physique, 1969, 30 (10), pp.849-855. 10.1051/jphys:019690030010084900 . jpa00206851

\section{HAL Id: jpa-00206851 https://hal.science/jpa-00206851}

Submitted on 1 Jan 1969

HAL is a multi-disciplinary open access archive for the deposit and dissemination of scientific research documents, whether they are published or not. The documents may come from teaching and research institutions in France or abroad, or from public or private research centers.
L'archive ouverte pluridisciplinaire HAL, est destinée au dépôt et à la diffusion de documents scientifiques de niveau recherche, publiés ou non, émanant des établissements d'enseignement et de recherche français ou étrangers, des laboratoires publics ou privés. 


\title{
ÉTUdE THÉORIQUE DES SPEGTRES OPTIQUES DES IONS $\mathrm{Pr}^{3+}$ DANS $\mathrm{AlLaO}_{3}$
}

\author{
Par N. PELLETIER-ALLARD et F. MARTIN-BRUNETIÈRE (1), \\ Laboratoire Aimé-Gotton, G.N.R.S. II, Faculté des Sciences, 9I-Orsay.
}

(Reçu le 27 mars 1969.)

\begin{abstract}
Résumé. - Les spectres d'absorption et de fluorescence de l'ion praséodyme dans l'aluminate de lanthane ont été interprétés dans l'approximation du champ cristallin, en considérant un ion de configuration fondamentale $4 f^{2}$ placé dans un site de symétrie $\mathrm{C}_{3 v}$. La méthode paramétrique a été utilisée avec traitement complet de l'hamiltonien du système. La complexité des spectres expérimentaux et le petit nombre de raies attribuées a priori avec certitude ont imposé la nécessité d'une étude très progressive qui a permis le calcul précis des niveaux de l'ion en fonction de 13 paramètres et l'attribution de l'ensemble des raies électroniques du spectre. L'écart quadratique moyen obtenu sur 42 niveaux expérimentaux est de $\pm 23 \mathrm{~cm}^{-1}$, ce qui signifie que la précision théorique sur l'ensemble de la configuration est $1 \%$.
\end{abstract}

Abstract. - The absorption and fluorescence spectra of praseodymium ion in $\mathrm{AlLaO}_{3}$ have been interpreted by use of crystal field theory on the fundamental configuration $4 f^{2}$ of an ion in $\mathrm{a}_{3 \mathrm{v}}$ site symmetry. The parametric method has been used and the hamiltonian of the system has been completely treated. The complexity of the experimental spectra and the small number of lines which can be assigned a priori with certainty have made necessary a study by successive approximations, which allowed the precise calculation of the levels of the ion as a function of 13 parameters and the assignment of all electronic lines of the system. The means square error obtained on 42 experimental levels is $\pm 23 \mathrm{~cm}^{-1}$, which indicates that the theoretical precision for the entire configuration is $1 \%$.

I. Introduction. - Les niveaux d'énergie de l'ion $\mathrm{Pr}^{3+}$ dans une matrice d'aluminate de lanthane ont été obtenus par étude des spectres d'absorption et de fluorescence [1]. Les spectres de la plupart des sels de terres rares sont composés de groupes de raies correspondant aux niveaux de l'ion libre dont la dégénérescence est partiellement levée par l'action de l'entourage de l'ion. Cette action est relativement faible, les électrons $4 f$ se trouvant protégés del'influence extérieure par les couches externes complètes $5 s^{2}$ et $5 p^{6}$.

Les spectres des sels de praséodyme ont été étudiés dans de nombreuses matrices. Après les premières recherches d'interprétation de niveaux cristallins du $\mathrm{Pr}^{3+}$ par Hellwege et Hellwege [2, 3] dans $\operatorname{Pr}_{2} \mathrm{Zn}_{3}\left(\mathrm{NO}_{3}\right)_{12}$, $24 \mathrm{H}_{2} \mathrm{O}$ et $\mathrm{Pr}_{2} \mathrm{Mg}_{3}\left(\mathrm{NO}_{3}\right)_{12}, 24 \mathrm{H}_{2} \mathrm{O}$, l'introduction des opérateurs équivalents par Stevens [4] a permis une analyse théorique des éthylsulfates de terres rares par Elliot et Stevens [5] et des nitrates doubles par Judd [6]. Les niveaux de $\mathrm{PrCl}_{3}$ identifiés par Dieke et Sarup [7] ont été interprétés théoriquement par Margolis [8] qui traite séparément, par une méthode de moindres carrés, les décompositions en multiplets et les décompositions dues au champ cristallin, et introduit, par un

(1) Actuellement : Laboratoire de Spectroscopie Atomique, Faculté des Sciences, 14-Caen. calcul au premier ordre, les interactions magnétiques. C'est également par traitement séparé des intégrales de Slater et du couplage spin-orbite, puis des effets relatifs au champ cristallin, que Gruber [9] a étudié le praséodyme dans des matrices d'éthylsulfate.

Ges différentes études ont montré la nécessité, pour une analyse rigoureuse du spectre d'un ion placé dans une matrice cristalline, de considérer simultanément les effets relatifs à l'ion lui-même et à son entourage. C'est dans ce but que l'interprétation des niveaux d'énergie de l'ion $\operatorname{Pr}^{3+}$ dans l'aluminate de lanthane, que nous proposons ici, a été faite par traitement complet de l'hamiltonien du système. Cette étude a été menée dans le cadre de l'approximation du champ cristallin, mais en introduisant l'ensemble des termes agissant sur les énergies dans chacune des deux matrices relatives aux symétries possibles pour les niveaux dans la structure considérée.

$\mathrm{AlLaO}_{3}$ a une structure rhomboédrique de type pérovskite déformé le long d'un axe ternaire du cube, et contenant deux fois la formule chimique par maille élémentaire. Le groupe spatial exact n'a pu être déterminé, et les dernières études cristallographiques donnent $\mathrm{R} 3 \mathrm{c}$ et $\mathrm{R} \overline{3} \mathrm{c}$ comme groupes possibles [10], l'indétermination provenant de l'incertitude des mesures. Dans la maille pérovskite idéale, l'ion terre rare 
est entouré de 8 ions $\mathrm{Al}^{3+}$ aux sommets d'un cube et de 12 ions $\mathrm{O}^{2-}$ aux milieux des arêtes, répartis en trois plans successifs perpendiculaires à un axe ternaire $A_{3}$. La déformation rhomboédrique se manifeste par une rotation de $+6^{\circ}$ et $-6^{\circ}$ des trois ions $\mathrm{O}^{2-}$ des plans extrêmes autour de $A_{3}$, entraînant des déplacements des 6 ions $\mathrm{O}^{2-}$ du plan médian qui contient aussi l'ion $\mathrm{La}^{3+}$.

Les résultats spectroscopiques ont montré que les ions $\mathrm{Pr}^{3+}$ se trouvent, en première approximation, dans des sites de symétrie ponctuelle $\mathrm{D}_{3}$ correspondant au groupe spatial $\mathrm{R} \overline{3} \mathrm{c}$. Ils ont mis de plus en évidence l'existence d'un champ de symétrie inférieure à la symétrie ternaire, mais les dédoublements observés étant de l'ordre de grandeur de la précision des mesures, l'approximation du champ ternaire a paru suffisante. Les calculs ont donc été faits dans le cas d'une symétrie d'ordre 3 correspondant au groupe $\mathrm{C}_{3 \mathrm{v}}$, isomorphe du groupe $\mathrm{D}_{3}$ mais présentant l'avantage de ne pas introduire de nombre imaginaire.

II. Méthodes de calcul et paramètres employés. Dans l'approximation du champ cristallin, l'hamiltonien $\mathscr{H}$ du système, en l'absence de champ magnétique externe, se met sous la forme :

$$
\mathscr{H}=\mathscr{H}_{4 f^{2}}+\mathscr{H}_{\text {crist }} .
$$

Dans cette expression, $\mathscr{H}_{4 f^{2}}$ est l'hamiltonien de l'ion libre et comprend l'énergie de configuration, l'interaction coulombienne et le couplage spin-orbite, les intensités de ces différents termes étant légèrement modifiées par la présence de l'entourage de l'ion.

$\mathscr{H}_{\text {crist }}$ représente la composante statique du champ cristallin. La théorie du champ cristallin suppose que l'interaction des électrons avec le milieu environnant est d'origine électrostatique, et que le potentiel électrique produit par les atomes entourant l'ion central possède la symétrie de l'arrangement de ces atomes. On montre alors que l'hamiltonien représentant le potentiel cristallin peut être mis sous la forme :

$$
\mathscr{H}_{\text {crist }}=A_{k}^{q}\left\langle r^{k}\right\rangle U_{q}^{(k)}
$$

les opérateurs $U_{q}^{(k)}$ définis par Racah sont proportionnels aux harmoniques sphériques $Y_{k q}$, solutions de l'équation de Laplace à laquelle obéit le potentiel; les coefficients $A_{k}^{q}\left\langle r^{k}\right\rangle$ représentent l'intensité du potentiel cristallin, $A_{k}^{q}$ dépendant uniquement de la structure du cristal et $\left\langle r^{k}\right\rangle$ étant la valeur moyenne de $r^{k}$ pour les électrons $4 f$.

La détermination des coefficients $A_{k}^{q}\left\langle r^{k}\right\rangle$ peut se faire soit paramétriquement, soit par calcul direct à partir des données structurales et des valeurs des fonctions radiales. Nous avons ici utilisé les deux méthodes et comparé les résultats obtenus. Dans la suite, nous noterons $B_{k}^{q}$ le paramètre $A_{k}^{q}\left\langle r^{k}\right\rangle$.

Dans le cas d'un ion de configuration fondamentale $4 f^{2}$ placé dans un site de symétrie $\mathrm{C}_{3 \mathrm{v}}$, les conditions imposées aux valeurs de $k$ et de $q$ par la symétrie ponctuelle du site des ions $\mathrm{Pr}^{3+}$ et les propriétés des opérateurs tensoriels limitent à six le nombre des coefficients entrant dans l'expression du potentiel, et l'hamiltonien cristallin s'écrit :

$$
\begin{aligned}
& \mathscr{H}_{\text {crist }}=B_{2}^{0} U_{0}^{(2)}+B_{4}^{0} U_{0}^{(4)}+B_{4}^{3}\left(U_{-3}^{(4)}-U_{3}^{(4)}\right) \\
& \quad+B_{6}^{0} U_{0}^{(6)}+B_{6}^{3}\left(U_{-3}^{(6)}-U_{3}^{(6)}\right)+B_{6}^{6}\left(U_{-6}^{(6)}+U_{6}^{(6)}\right) .
\end{aligned}
$$

Les 6 paramètres ainsi introduits s'ajoutent aux 7 paramètres relatifs aux seuls électrons $f$ et qui se trouvent dans l'expression due à Racah [11, 12] :

$$
\begin{aligned}
\mathscr{H}_{4 f^{2}}= & E_{0} e_{0}+E_{1} e_{1}+E_{2} e_{2}+E_{3} e_{3} \\
& +\alpha L(L+1)+12 \gamma G\left(G_{2}\right)-42 \zeta W^{(11) 0}(f, f)
\end{aligned}
$$

(le terme $10 \beta G\left(R_{7}\right)$ a été écarté de cet hamiltonien car il ne fournit aucune contribution aux niveaux observés, si ce n'est un déplacement général absorbé par $E_{0}$ ).

Les spectres obtenus expérimentalement comportant un grand nombre de raies de vibration et d'interaction, peu de raies correspondant à des transitions électroniques pures avaient pu être attribuées a priori.

L'étude a, par conséquent, été menée de façon progressive. Nous avons, dans un premier temps, traité de façon indépendante les termes relatifs à l'ion libre et ceux relatifs au champ cristallin, ce qui nous a donné des valeurs approximatives des paramètres. Ces valeurs ont ensuite été introduites dans le traitement total de l'hamiltonien avec des hypothèses variables quant aux niveaux pour lesquels l'attribution était douteuse. Par approximations successives, nous avons ainsi pu attribuer précisément l'ensemble des raies électroniques du spectre et déterminer les valeurs des 13 paramètres entrant en jeu de façon suffisamment exacte, pour rendre compte du spectre de manière satisfaisante.

L'étude a nécessité, après le calcul de tous les éléments de matrice, un grand nombre de diagonalisations, chacune d'elles suivie d'une série d'optimisations de paramètres par la méthode des moindres carrés. Tous ces calculs ont été effectués sur la calculatrice Univac 1108 de la Faculté des Sciences d'Orsay, à l'aide de programmes mis au point au Laboratoire Aimé-Cotton $[13,14,15,16]$.

III. Première détermination des paramètres. L'application de la théorie des groupes finis à un ion de configuration $4 f^{2}$ placé dans un site de symétrie $\mathrm{C}_{3 \mathrm{v}}$ montre que les 91 sous-niveaux de la configuration se groupent en 30 niveaux de nombre quantique $M_{J}= \pm 1$ modulo 3 doublement dégénérés (niveaux $E$ dans la notation de Placzek), et 31 niveaux de nombre quantique $M_{J}=0$ modulo 3 non dégénérés (niveaux $A$ ). La symétrie à laquelle appartient chaque niveau est déterminée par la polarisation des raies et par leurs spectres Zeeman et Faraday [17].

L'étude expérimentale avait permis l'attribution sûre de 11 raies seulement, et le tableau I a donne les sous-niveaux correspondants, sur lesquels les calculs 
ont été menés dès le début. Cependant, un certain nombre de sous-niveaux, dont la symétrie n'avait pas été trouvée expérimentalement, ont pu être attribués à des niveaux déterminés de l'ion libre (tableau I b) et, par conséquent, être utilisés dans l'étude en champ nul.

\section{TABLEAU I}

a

Sous-niveaux $A$ Sous-niveaux $E$

\begin{tabular}{|c|c|c|}
\hline & $\begin{array}{c}\left(\mathrm{cm}^{-1}\right) \\
-\end{array}$ & $\begin{array}{c}\left(\mathrm{cm}^{-1}\right) \\
-\end{array}$ \\
\hline${ }^{3} H_{4}$ & & $\left\{\begin{array}{r}0 \\
179 \\
229\end{array}\right.$ \\
\hline${ }^{1} D_{2}$ & 16832 & $\left\{\begin{array}{l}16694 \\
16792\end{array}\right.$ \\
\hline${ }^{3} P_{0}$ & 20594 & \\
\hline${ }^{3} P_{1}$ & 21163 & 21201 \\
\hline${ }^{3} P_{2}$ & 22490 & $\left\{\begin{array}{l}22348 \\
22461\end{array}\right.$ \\
\hline
\end{tabular}

b

Centres de gravité Des niveaux $\left(\mathrm{cm}^{-1}\right)$

$\begin{array}{lr}{ }^{3} H_{4} & 180 \\ { }^{3} H_{5} & 2270 \\ { }^{1} G_{4} & 10070 \\ { }^{1} D_{2} & 16773 \\ { }^{3} P_{0} & 20594 \\ { }^{1} I_{6} & 21520 \\ { }^{3} P_{1} & 21182 \\ { }^{3} P_{2} & 22478\end{array}$

1. DÉtermination des PARAMÈtres RElAtifs A L'ION. - La connaissance de l'ensemble des sous-niveaux issus de ${ }^{3} \mathrm{P}$ a permis la détermination de la constante $\zeta$ de couplage spin-orbite.

Le centre de gravité des niveaux en champ nul a été calculé d'après les données expérimentales alors connues pour les 5 termes ${ }^{3} H,{ }^{1} G_{4},{ }^{1} D_{2},{ }^{3} P$ et ${ }^{1} I_{6}$. A partir des énergies de ces centres de gravité, nous avons tenté d'obtenir une valeur approximative des paramètres $E_{0}, E_{1}, E_{2}, E_{3}$ par un calcul de moindres carrés. Le paramètre $\alpha$ relatif aux effets d'une théorie linéaire de l'interaction de configuration a également été introduit dans ce premier calcul, mais afin de conserver un nombre de paramètres variables inférieur au nombre des données expérimentales, nous avons fixé sa grandeur à celle trouvée par Sugar pour l'ion libre [18]. Le paramètre $\gamma$ n'a été introduit que dans le traitement complet de l'hamiltonien. Ce travail ayant été commencé avant que ne soit publiée sa valeur dans l'interprétation des niveaux du praséodyme [19], nous avons introduit dans nos calculs celle obtenue pour le cour $f^{2}$ de la configuration $f^{2}(d+s)$ de Pr III [20].

L'ensemble des résultats obtenus a été porté dans le tableau II a, ainsi que l'écart quadratique moyen au sens de Racah $\overline{\Delta E}$.

TABLEAU II

a

VALEURS DES PARAMÈTRES RELATIFS A L'ION $\left(\mathrm{en}^{\mathrm{cm}}{ }^{-1}\right)$

$\begin{array}{cc}E_{0} & 6256 \pm 4 \\ E_{1} & 4855 \pm 3 \\ E_{2} & 20,9 \pm 0,1 \\ E_{3} & 463 \pm 0,2 \\ \alpha & 19 \text { fixé } \\ \zeta & 755 \pm 2 \\ \overline{\Delta E} & \pm 26 \\ \gamma & -56\end{array}$

b

VALEURS DES PARAMÈTRES RELATIFS AU GHAMP GRISTALLIN (en $\mathrm{cm}^{-1}$ )

$\begin{array}{rr}B_{2}^{0} & 178 \\ B_{4}^{0} & 442 \\ B_{4}^{3} & -\quad 460 \\ B_{6}^{0} & 1600 \\ B_{6}^{3} & 1000 \\ B_{6}^{6} & 1800\end{array}$

2. DÉtermination Des PARAmÈtres RElatifs AU GHAMP GRISTALLIN. - Les éléments de matrice, diagonaux entre deux états $\left|\gamma S L J M_{J}\right\rangle$, des opérateurs $U_{q}^{(k)}$ étant nuls par définition pour des valeurs de $k$ supérieures à $2 L$, les niveaux ${ }^{3} P$ dépendent, au point de vue cristallin, du seul paramètre $B_{2}^{0}$, et les niveaux ${ }^{1} D_{2}$ ne dépendent que de $B_{2}^{0}, B_{4}^{0}$ et $B_{4}^{3}$. Il est alors facile, dans l'approximation du premier ordre, c'est-à-dire en considérant les termes comme indépendants, de tirer les valeurs de ces paramètres des séparations observées pour les sous-niveaux correspondants.

Nous avons choisi de calculer $B_{2}^{0}$ à partir de ${ }^{3} P_{1}$, seul niveau de la configuration dont le nombre quantique $J$ soit égal à 1 , ce qui permet de penser que la différence d'énergie entre les deux sous-niveaux ne sera pas modifiée dans le traitement complet de l'hamiltonien. Les valeurs de $B_{4}^{0}$ et $B_{4}^{3}$ ont été obtenues à partir des séparations entre le sous-niveau $A$ et les deux sous-niveaux $E$ de ${ }^{1} D_{2}$; ce calcul est forcément très approximatif car on peut prévoir que ${ }^{1} D_{2}$ sera très mélangé aux autres niveaux, à ${ }^{3} P_{2}$ et ${ }^{3} F_{2}$ ne particulier.

Une approche des valeurs des paramètres $B_{6}^{q}$ ne pouvait être faite aussi simplement, et nous avons été 
amenés à tracer des courbes représentant les énergies des niveaux lorsqu'on faisait varier ces paramètres. Ces courbes ont mis en évidence que la symétrie $E \mathrm{du}$ sous-niveau de base entraînait pour $B_{6}^{0}$ une valeur positive et relativement élevée. La comparaison des résultats obtenus par variation d'un paramètre $B_{6}^{q}$ en fixant les deux autres a montré que $B_{6}^{0}, B_{6}^{3}$ et $B_{6}^{6}$ étaient du même ordre de grandeur et a permis de choisir les valeurs de départ.

Les grandeurs ainsi obtenues pour les paramètres du champ cristallin étant destinées à être introduites dans une étude tenant compte de l'ensemble des interactions, il n'a pas paru intéressant de faire un calcul de moindres carrés préalable sur ces paramètres en considérant, comme l'ont fait certains auteurs, les énergies de chaque terme de l'ion libre comme des paramètres indépendants librement ajustables aux résultats expérimentaux.

IV. Résultats. - Le traitement total de l'hamiltonien $\mathscr{H}$ du système a consisté à tenir compte simultanément de l'ensemble des interactions considérées, c'est-à-dire à introduire les treize paramètres dans chacun des deux déterminants séculaires donnant les énergies des niveaux d'un ion $\operatorname{Pr}^{3+}$ dans une matrice cristalline de symétrie $\mathrm{C}_{3 \mathrm{v}}$. Les ordres de grandeur des paramètres étaient connus, mais leur nombre était supérieur à celui des données expérimentales qu'il était possible d'introduire d'emblée dans le calcul de moindres carrés. Il a donc été nécessaire de travailler par approximations successives. Nous avons commencé par fixer ceux des paramètres dont les variations avaient les influences les plus faibles sur les énergies des niveaux, ou dont la détermination paraissait suffisamment bonne dans les estimations préliminaires. Ainsi la suppression, en tant que paramètres variables, de $E_{2}$, $E_{3}, \zeta, \alpha, B_{2}^{0}$, autorisait un calcul de moindres carrés puisque nous étions ramenés à 12 niveaux et 7 paramètres. Les énergies ainsi obtenues pour l'ensemble des niveaux de la configuration ont permis l'attribution de quelques sous-niveaux supplémentaires, dont les valeurs ont pu être ensuite incluses dans les moindres carrés. Une telle démarche, répétée plusieurs fois, et permettant chaque fois d'introduire un nombre plus grand de paramètres et de niveaux, a finalement mené, d'une part à la détermination de l'ensemble des paramètres, d'autre part à l'attribution précise de toutes les raies électroniques mesurées dans le spectre à des transitions entre sous-niveaux d'énergie bien définis.

Sur le tableau III ont été portées les valeurs définitives ainsi obtenues pour les treize paramètres. Elles ont été calculées par variation simultanée de 12 d'entre eux, 42 niveaux expérimentaux ayant été introduits qui appartiennent à tous les termes à l'exception $\mathrm{de}^{1} S_{0}$. Seule la valeur de $B_{2}^{0}$ a été fixée de telle manière que l'écart calculé entre les deux sous-niveaux de ${ }^{3} P_{1}$, qui dépend à 99,7\% de ce seul paramètre, soit égal à l'écart observé. Il faut d'ailleurs noter que $B_{2}^{0}$ intro-
TABLEAU III

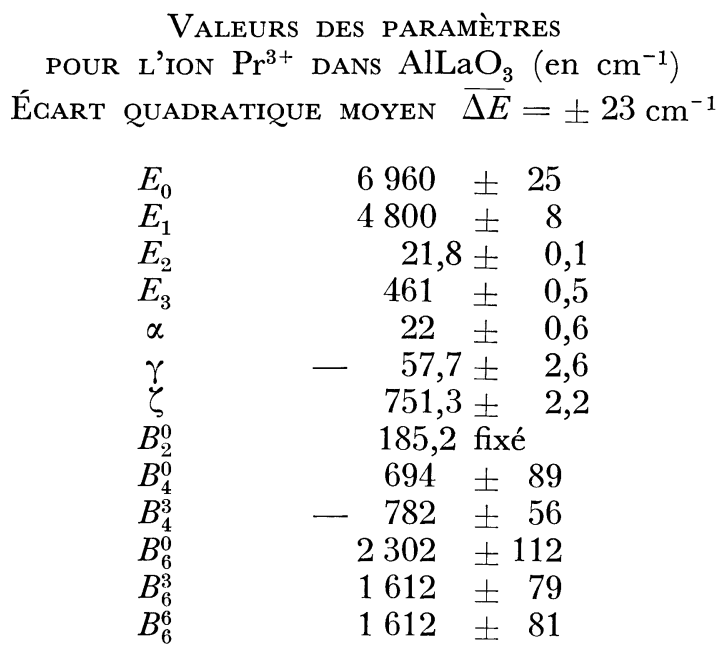

duit dans les moindres carrés comme paramètre variable se fixe très mal $(105 \pm 69)$ tout en n'améliorant en rien l'ensemble des résultats.

L'écart quadratique moyen obtenu dans ce calcul est égal à $\pm 23 \mathrm{~cm}^{-1}$. Il peut être jugé comme très faible si l'on considère qu'il est relatif à l'ensemble des termes agissant sur les énergies des niveaux et qu'il a été calculé sur 42 niveaux expérimentaux. Les valeurs exactes des énergies observées et calculées ont été portées dans le tableau IV, et la figure permet de visualiser la comparaison entre valeurs calculées et valeurs observées; les niveaux ont été groupés par termes bien que la configuration ait été calculée dans son ensemble.

V. Calcul direct des paramètres du champ cristallin. - Le calcul des coefficients $A_{k}^{q}\left\langle r^{k}\right\rangle$ du champ cristallin peut se faire également par une méthode a priori, à partir du modèle géométrique que forment les ions créant le potentiel, et des valeurs moyennes de $r^{k}$ de l'ion terre rare. La difficulté d'appliquer rigoureusement cette méthode réside dans le fait que :

1. Les valeurs des intégrales radiales sont modifiées par le recouvrement des fonctions d'onde de l'ion terre rare par celles des ions voisins.

2. Il est nécessaire de tenir compte de l'ensemble des ions du cristal agissant sur l'ion terre rare et de faire intervenir les interactions multipolaires.

Les valeurs trouvées pour $\left\langle r^{k}\right\rangle$ en supposant l'ion libre sont loin d'être satisfaisantes; les valeurs des coefficients $A_{k}^{q}$, au contraire, peuvent être relativement bien approchées par un calcul théorique négligeant les ions autres que les plus proches voisins et utilisant le simple modèle ionique. Il est par conséquent possible d'obtenir des rapports $A_{k}^{q}\left\langle r^{k}\right\rangle \mid A_{k}^{q^{\prime}}\left\langle r^{k}\right\rangle$ assez voisins des rapports trouvés par voie paramétrique. Nous avons 
TABLEAU IV

\begin{tabular}{|c|c|c|c|c|c|c|c|}
\hline Niveaux & $\begin{array}{c}\text { ÉNERGIES } \\
\text { OBSERVÉES } \\
\left(\mathrm{cm}^{-1}\right)\end{array}$ & $\begin{array}{c}\text { ÉNERGIES } \\
\text { GALGULÉES } \\
\left(\mathrm{cm}^{-1}\right)\end{array}$ & $\mathrm{O}-\mathrm{C}$ & Niveaux & $\begin{array}{c}\text { ÉNERGIES } \\
\text { OBSERVÉES } \\
\left(\mathrm{cm}^{-1}\right)\end{array}$ & $\begin{array}{c}\text { ÉNERGIES } \\
\text { GALCULÉES } \\
\left(\mathrm{cm}^{-1}\right)\end{array}$ & $\mathrm{O}-\mathrm{C}$ \\
\hline - & - & - & - & - & - & - & - \\
\hline${ }^{3} H_{4}, E$ & 0 & -9 & 9 & ${ }^{3} F_{4}, A$ & & 6905 & \\
\hline${ }^{3} H_{4}, A$ & 87 & 145 & -58 & ${ }^{3} F_{4}, E$ & 6919 & 6907 & 12 \\
\hline${ }^{3} H_{4}, E$ & 179 & 188 & -9 & ${ }^{3} F_{4}, A$ & & 7017 & \\
\hline${ }^{3} H_{4}, A$ & 229 & 228 & 1 & ${ }^{3} F_{4}, E$ & & 7109 & \\
\hline${ }^{3} H_{4}, E$ & 229 & 237 & -8 & ${ }^{3} F_{4}, A$ & 7099 & 7126 & -27 \\
\hline${ }^{3} H_{4}, A$ & & 755 & & ${ }^{3} F_{4}, E$ & & 7128 & \\
\hline${ }^{3} H_{5}, E$ & 2197 & 2183 & 14 & ${ }^{1} G_{4}, A$ & & 9634 & \\
\hline${ }^{3} H_{5}, A$ & & 2236 & & ${ }^{1} G_{4}, E$ & & 9642 & \\
\hline${ }^{3} H_{5}, E$ & 2234 & 2238 & -4 & ${ }^{1} G_{4}, A$ & 9695 & 9683 & 12 \\
\hline${ }^{3} H_{5}, A$ & 2267 & 2255 & 12 & ${ }^{1} G_{4}, E$ & 10071 & 10078 & -7 \\
\hline${ }^{3} H_{5}, E$ & 2308 & 2300 & 8 & ${ }^{1} G_{4}, A$ & & 10118 & \\
\hline${ }^{3} H_{5}, A$ & & 2647 & & ${ }^{1} G_{4}, E$ & 10438 & 10430 & 8 \\
\hline${ }^{3} H_{5}, E$ & & 2660 & & ${ }^{1} D_{2}, E$ & 16694 & 16681 & 13 \\
\hline${ }^{3} H_{6}, A$ & 4087 & 4104 & -17 & ${ }^{1} D_{2}, E$ & 16792 & 16789 & 3 \\
\hline${ }^{3} H_{6}, A$ & 4276 & 4264 & 12 & ${ }^{1} D_{2}, A$ & 16832 & 16837 & -5 \\
\hline${ }^{3} H_{6}, E$ & 4266 & 4276 & -10 & ${ }^{3} P_{0}, A$ & 20594 & 20556 & 38 \\
\hline${ }^{3} H_{6}, A$ & 4337 & 4334 & 3 & ${ }^{3} P_{1}, A$ & 21163 & 21136 & 27 \\
\hline${ }^{3} H_{6}, E$ & 4384 & 4373 & 11 & ${ }^{3} P_{1}, E$ & 21202 & 21175 & 27 \\
\hline${ }^{3} H_{6}, A$ & 4438 & 4452 & -14 & ${ }^{1} I_{6}, \quad A$ & & 21224 & \\
\hline${ }^{3} H_{6}, E$ & 4844 & 4833 & 11 & ${ }^{1} I_{6}, \quad E$ & 21277 & 21263 & 14 \\
\hline${ }^{3} H_{6}, E$ & 4895 & 4851 & 44 & ${ }^{1} I_{6}, E$ & 21306 & 21314 & -8 \\
\hline${ }^{3} H_{6}, A$ & & 4870 & & ${ }^{1} I_{6}, \quad A$ & & 21560 & \\
\hline${ }^{3} F_{2}, E$ & 5248 & 5245 & 3 & ${ }^{1} I_{6}, \quad E$ & & 21617 & \\
\hline${ }^{3} F_{2}, A$ & 5275 & 5256 & 19 & ${ }^{1} I_{6}, E$ & & 21639 & \\
\hline${ }^{3} F_{2}, E$ & 5285 & 5281 & 4 & ${ }^{1} I_{6}, \quad A$ & & 21640 & \\
\hline${ }^{3} F_{3}, A$ & 6394 & 6391 & 3 & ${ }^{1} I_{6}, \quad A$ & & 21728 & \\
\hline${ }^{3} F_{3}, E$ & 6504 & 6506 & -2 & ${ }^{1} I_{6}, \quad A$ & & 21757 & \\
\hline${ }^{3} F_{3}, A$ & 6496 & 6508 & -12 & ${ }^{3} P_{2}, E$ & 22348 & 22390 & -42 \\
\hline${ }^{3} F_{3}, E$ & 6698 & 6698 & 0 & ${ }^{3} P_{2}, E$ & 22461 & 22488 & -27 \\
\hline${ }^{3} F_{3}, A$ & 6685 & 6701 & -16 & $\begin{array}{l}{ }^{3} P_{2}, A \\
{ }^{1} S_{0}, A\end{array}$ & 22490 & $\begin{array}{l}22529 \\
50419\end{array}$ & -39 \\
\hline
\end{tabular}

donc calculé les 6 quantités (pour les notations, voir [6]) :

$$
\frac{4 \pi}{2 k+1} \sum_{j} \frac{g_{j} e^{2}}{\rho_{j}^{k+1}} \mathrm{Y}_{k q}\left(\alpha_{j}, \beta_{j}\right)
$$

en introduisant les 12 ions $\mathrm{O}^{2-}$ et les 8 ions $\mathrm{Al}^{3+}$ les plus proches d'un ion terre rare, et en tenant compte des déformations de la maille relativement à la maille pérovskite.

Cependant, la complexité de la structure ne permettait pas de considérer comme suffisante a priori l'approximation choisie, et nous n'avons pas introduit dans les calculs paramétriques les valeurs ainsi déterminées des rapports $B_{k}^{q} / B_{k}^{q^{\prime}}$. 


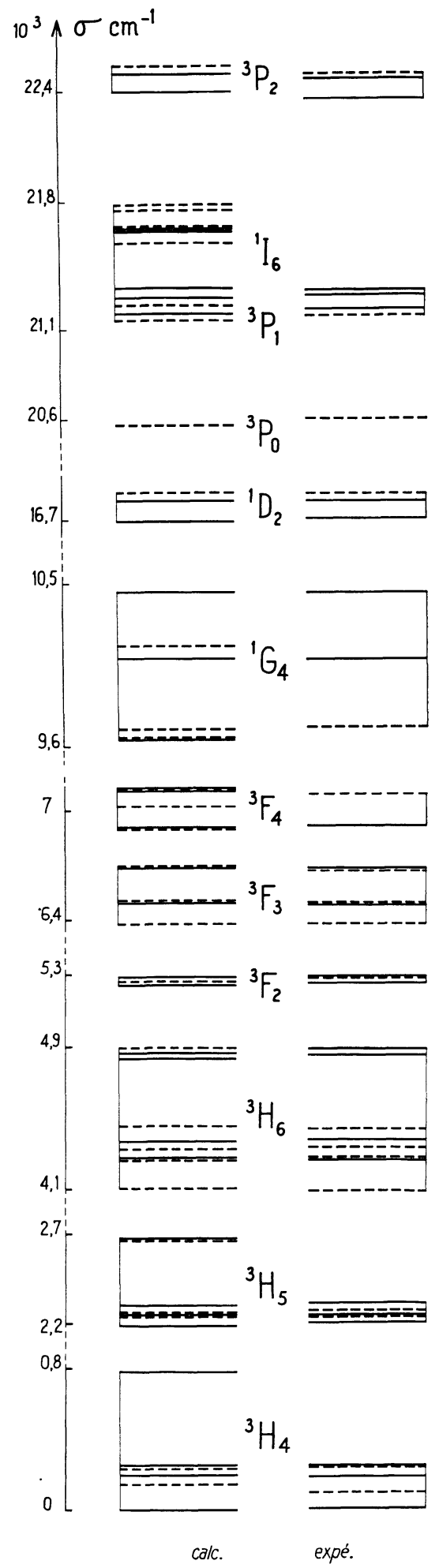

FIG. 1. - Niveaux d'énergie, calculés et expérimentaux, du praséodyme dans l'aluminate de lanthane :

- niveaux $E$.

- - niveaux $A$.
Les résultats obtenus pour les coefficients $B_{6}^{q}$ donnent les valeurs suivantes des rapports :

$$
B_{6}^{3} / B_{6}^{0}=0,57 ; \quad B_{6}^{6} / B_{6}^{0}=0,66 .
$$

Les deux mêmes rapports obtenus par la méthode paramétrique sont égaux à 0,70 .

Le calcul fait pour les coefficients $B_{4}^{q}$ conduit à l'évaluation du rapport d'un numérateur de l'ordre de grandeur des incertitudes, sur un dénominateur également petit, donc à une indétermination numérique sur le signe comme sur la valeur; ceci signifie que $B_{4}^{3} / B_{4}^{0}$ est très sensible à une légère variation de structure. Et, effectivement, nous avons remarqué, au cours de nos approximations successives, la grande variabilité de ce rapport lors de faibles variations des $B_{6}^{q}$; notons que la valeur 1,1 à laquelle il s'est fixé (tableau III) reste en bon accord avec celle que nous avions introduite à partir des séparations entre les sous-niveaux $E$ de ${ }^{1} D_{2}$ (tableau II).

En résumé, les résultats comparés du calcul des rapports des $B_{k}^{q}$ de mêmes valeurs de $k$, obtenus par méthodes structurales et paramétriques, sont cohérents pour $B_{4}^{3} / B_{4}^{0}$ et satisfaisants pour $B_{6}^{6} / B_{6}^{0}$. L'accord pour $B_{6}^{3} / B_{6}^{0}$ est moins bon, ce qui s'explique par le fait que ce rapport est de beaucoup le plus sensible à une incertitude de position des oxygènes; l'erreur reste cependant inférieure à $20 \%$, ce qui la place dans les limites généralement obtenues.

VI. Conclusion. - Le calcul des niveaux d'énergie d'un ion de configuration fondamentale $4 f^{2}$ placé dans un entourage de symétrie $\mathrm{C}_{3 \mathrm{v}}$ a permis d'interpréter les spectres d'absorption et de fluorescence de cristaux d'aluminate de lanthane dopés au praséodyme. Cette étude a été faite dans le cadre de la théorie du champ cristallin, et nous a amenés à déterminer la valeur des paramètres définissant l'intensité de chacun des termes de l'hamiltonien du système. La partie relative à l'ion $\mathrm{Pr}^{3+}$, dans laquelle on a tenu compte des perturbations apportées par les configurations supérieures, a été calculée indépendamment de celle relative à l'entourage cristallin; les valeurs obtenues ont servi de point de départ à une démarche progressive grâce à laquelle toutes les raies observées ont été attribuées à des transitions entre niveaux bien définis.

Un calcul exact a alors pu être fait car, en traitant simultanément tous les effets agissant sur la configuration, nous n'avons laissé place à aucune approximation dans le cadre de la théorie choisie. Les résultats ainsi obtenus semblent satisfaisants puisque l'erreur relative entre données expérimentales et valeurs calculées n'est que de $1 \%$ oo sur l'ensemble de la configuration. L'intérêt de la méthode utilisée a également été de mettre en évidence l'influence réciproque des effets dus à l'ion d'une part, et à son entourage d'autre part; nous avons ainsi montré que son emploi était nécessaire à l'analyse rigoureuse d'un ion placé dans une matrice cristalline. 


\section{BIBLIOGRAPHIE}

[1] MARTin-BRUNETIÈRE (F.), Étude expérimentale des spectres optiques des ions $\mathrm{Pr}^{3+}$ dans $\mathrm{AlLaO}_{3}$.

[2] Hel, weGE (A. M.) et HEILWEGE (K. H.), Z. Physik, 1951, 130, 549 .

[3] Hellwege (A. M.) et Heiliwege (K. H.), Z. Physik, 1953, 135, 92.

[4] Stenens (K. W. H.), Proc. Phys. Soc. A, 1952, 65, 209.

[5] Elifiot (R. J.) et Stenvens (K. W. H.), Proc. Roy. Soc. $A, 1952,215,437$.

[6] JUdD (B. R.), Proc. Roy. Soc. A, 1955, 232, 458.

[7] Dieke (G. H.) et SARup (R.), J. Chem. Phys., 1958, 29, 741.

[8] Margolis (J. S.), J. Chem. Phys., 1961, 35, 1367.

[9] Gruber (J. B.), J. Chem. Phys., 1963, 38, 946.

[10] Zelver (C.), Thèse, Paris, 1967, et références citées.

[11] Racah (G.), Phys. Rev., 1949, 76, 1352.

[12] Racah (G.), Phys. Rev., 1952, 85, 381.
[13] Bordarier (Y.), Carlier (A.) et NGuyen-NGocChan, Programme Agenac de calcul de formules suivant l'algèbre de Racah, Brochure en préparation.

[14] Bordarier (Y.), Programme Assac de regroupement des résultats d'Agenac, Brochure en préparation.

[15] Bordarier (Y.) et DAGoury (P.), Programme Diagac de diagonalisation et de calcul des dérivées des énergies et des g, Orsay, 1968.

[16] BORDARIER (Y.), Programme Gramac d'optimisation des paramètres par moindres carrés, Brochure en préparation.

[17] Martin-Brunetière (F.) et Pelletiter-Alitard (N.), C. R. Acad. Sci. (à paraître).

Boccara (A. C.), Briat (B.) et FERRE (J.) (à paraître).

[18] Sugar (J.), J. Opt. Soc. Amer., 1963, 53, 831.

[19] Judd (B. R.), Crosswhite (H. M.) et Crosswhite (H.), Phys. Rev., 1968, 169, 130.

[20] Feneuilie (S.) et Pelletier-Alilard (N.), Physica, 1968, 40, 347. 